\title{
Linear Parameter-Varying Identification of the EMG-Force Relationship of the Human Arm
}

\author{
Mattia PESENTI ${ }^{1}$, Ziad ALKHOURY ${ }^{2}$, Maciej BEDNARCZYK ${ }^{2}$, Hassan OMRAN ${ }^{2}$ and Bernard BAYLE ${ }^{2}$ \\ ${ }^{1}$ Department of Information and Bioengineering, Politecnico di Milano, Milan (IT) \\ ${ }^{2}$ ICube Laboratory, University of Strasbourg, Strasbourg (FR)
}

\begin{abstract}
In this paper, we present a novel identification approach to model the EMG-Force relationship of the human arm, reduced to a single degree of freedom (1-DoF) for simplicity. Specifically, we exploit the Linear Parameter Varying (LPV) framework. The inputs of the model are the electromyographic (EMG) signals acquired on two muscles of the upper arm, biceps brachii and triceps brachii, and two muscles of the forearm, brachioradialis and flexor carpi radialis. The output of the model is the force produced at the hand actuating the elbow. Because of the position-dependency of the system, the elbow angle is used as scheduling signal for the LPV model. Accurate modeling of the human arm with this approach opens new possibilities in terms of robot control for physical HumanRobot Interaction and rehabilitation robotics.
\end{abstract}

Index Terms-Human modeling, system identification, biomedical signal processing, electromyography

\section{INTRODUCTION}

Skin electromyography (EMG) allows to acquire the electrical activity that is responsible of muscular contractions. This technique has become essential in a wide range of applications, as research on the human neuromotor mechanisms, clinical diagnostic methods [1] and neuro-prosthesis. Robotic rehabilitation devices show great potential when treating neurological injuries [2,3], such as strokes or spinal cord injuries, as the treatment involves repetitive movements. Clinical studies show the increase of patient recovery when the robot provides active aid by physical interaction [4, 5]. With the aim of improving the rehabilitation outcome, it would be possible to integrate an EMG-based model of the human limb into the robot control strategy.

EMG-driven models are developed considering as input the electromyographic signals acquired on the muscles of interest. The problem consists in determining the force developed by a set of muscles from the corresponding EMG signals. The EMG-Force relationship has been under research with two different approaches. The first one consists in phenomenological modeling by exploiting mathematical equations describing the neuromuscular system, such as the Hill model [6]. This modeling approach is highly nonlinear and depends on several subject-specific parameters. Even though optimization techniques have been proven to improve the fitting of these parameters [7], the model structure remains of high complexity and nonlinearity. The second approach, used in this work, avoids the drawbacks of the first one and is based on black-box system identification. More precisely, the model parameters are identified directly from input/output datasets. Several works have considered the black-box approach to model the EMG-Force relationship. In [8], a nonlinear dynamic polynomial model was proposed to describe the EMG-Torque relationship around the elbow. Other works showed that parallel cascade Wiener models can perform well in the identification of the EMGForce relationship considering both constant-posture trials [9] and free hand motions [10]. Here, the elbow angle of the subject has been included as additional input in order to identify the dependency of the system on the geometric configuration of the arm. Indeed, the shape of the EMGForce relationship scales depending on the elbow angle [11]. This modeling approach was extended also to the case of free hand motions [10]: releasing the constant-posture constraint led to a dramatic decrease of the modeling accuracy.

The presented black-box models are still highly nonlinear and computationally complex, thus being unsuitable for the integration within the control system of rehabilitation robots. Moreover, the accuracy of the presented models is generally limited to the quasi-static case (isometric contractions), while the performance decreased considering dynamic contractions.

Linear Parameter Varying (LPV) modeling has been recently applied to study the human neuromusculoskeletal system. Indeed, the LPV approach was exploited to model the nonlinearity (due to a position dependency) of the joint impedance, as for the case of the wrist [12] and the ankle [13]. The accuracy reported in these works serves as motivation to extend the LPV approach to the EMG-Force relationship of the 1-DoF human arm.

The present paper is structured as follows. In Section II, we introduce the class of LPV models. Then, in Section III, we describe the experimental setup. In Section IV, we present the main results, which are discussed in Section V. Finally, in Section VI, we conclude the paper.

\section{Preliminaries}

The Linear Parameter Varying (LPV) representation provides a framework to describe certain classes of nonlinear systems. Many tools dedicated to Linear Time-Invariant (LTI) systems can be extended to the LPV framework. Indeed, LPV models can be considered as an extension of LTI models. The practical advantage of an LPV model is that its input/output relationship is linear, while the model itself is capable of describing nonlinear and time-varying systems. 


\section{A. LPV Models}

A discrete-time, state-space LPV model is defined as follows

$$
\begin{aligned}
x(k+1) & =A(q(k)) x(k)+B(q(k)) u(k) \\
y(k) & =C(q(k)) x(k)+D(q(k)) u(k)
\end{aligned}
$$

where $x(k) \in \mathbb{R}^{n}$ is the state vector, $u(k) \in \mathbb{R}^{p}$ is the input vector and $y(k) \in \mathbb{R}^{m}$ is the output vector, all at time instant $k$. The order of the LPV model $(n)$ is the dimension of its state vector, while the integers $p, m$ represent the input and output dimensions, respectively. The state matrix $A(q(k)) \in \mathbb{R}^{n \times n}$, input matrix $B(q(k)) \in$ $\mathbb{R}^{n \times p}$, output matrix $C(q(k)) \in \mathbb{R}^{m \times n}$ and feed-through matrix $D(q(k)) \in \mathbb{R}^{m \times p}$ are all dependent on an external signal $q(k)$, which is usually called scheduling signal. In this work, we consider the subclass of Affine LPV (ALPV) models $[14,15]$. In this representation, the model matrices depend in an affine way on the scheduling signal, namely

$$
A(q(k))=A_{0}+A_{1} q(k)
$$

and similarly for $B(\cdot)$ and $C(\cdot)$. Without loss of generality, the feed-through term is considered to be zero, i.e. $D(\cdot)=0$. We focus on the ALPV representation for two reasons: first, the realization theory for this subclass of LPV models is already mature $[14,15]$; in addition, the control theory $[16$, 17] dedicated to ALPV models is well-developed. Moreover, many of the tools developed for the LTI case are extended to the LPV framework. This means that some LPV identification methods directly rely on identification tools developed for LTI models.

\section{B. LPV Identification}

LPV system identification can be divided into two main approaches based on the experiment design, namely the local and the global identification approaches [18]. The global approach $[19,20]$ requires performing only one experiment on the system, in which the input and the scheduling signals are excited in order to capture all the dynamics of the system. This is a natural way for obtaining datasets that describe the real system. In contrast, the local approach identifies multiple LTI models (usually called frozen LTI models) for multiple, constant scheduling signals. Then, the parameterdependent LPV model is fitted to the system matrices of the frozen LTI models using regressors formed from the scheduling signal $q[21,22]$. The local approach can be teared down to the following three steps.

1) Identification of linear state-space models for several values of the scheduling parameter space.

2) Transformation of the obtained LTI models to a consistent state-space representation [17].

3) Interpolation of the state-space matrices of the local models with respect to the scheduling signal in order to obtain the LPV model.

It is well-known that the local approach suffers from a systematic modeling error. However, this error is bounded and its bound depends on the speed of change of the scheduling signal [23]. On the other hand, the local approach is more common for two reasons: it is more practical in terms of experimental design and it benefits of the well-developed theory of LTI systems identification.

Considering the LPV model here presented, the input of the system is a set of EMG signals, the output is the measured force, while the scheduling signal is a quantity proportional to the elbow angle of the subject (see §III).

\section{EXPERIMENTAL SETUP}

In our setup, input/output data are acquired while the user interacts with a collaborative robot, the KUKA LBR iiwa. EMG and force data are synchronously acquired and stored to be processed offline. Black-box identification methods are then used to identify a model mapping the muscular activation signals into the force developed by the arm.

The joint configuration of the robot is set in order to kinematically couple the human elbow angle with the elbow joint of the robot, as shown in Fig 1. In this way, the user can rest their arm on the robot while standing next to it. Both the standing point and the arm resting point are marked for repeatability. This setup permits to achieve weight compensation of the arm without the use of any belts or braces. Moreover, the proportionality between the elbow angle of the user and the elbow joint of the robot allows to easily measure and vary the human elbow angle. In this way, the experimental setup allows to measure the interaction forces between the user and the robot in two modes. First, by fixing the robotic arm at a certain position (i.e. at a fixed angle), we can model the dynamics of the 1-DoF human arm in a constant-posture setting. This type of experiment is called local trial and it is used in the local approach for LPV identification. Second, we can apply a movement profile to the elbow joint of the robotic arm, which would lead to a time-varying elbow angle profile in the horizontal plane. This experiment is called hereafter global trial and results in datasets which are suitable for the global identification approach. During the experiments, the subjects are asked to stand still, maintaining a constant body posture, and focus on actuating only the elbow joint to generate force, while keeping the wrist at a fixed angle.

The EMG signals, which represent the input of the system, are acquired using the Delsys Trigno Wireless System (Delsys Inc) with a sampling rate of $2000 \mathrm{~Hz}$. Four muscles of the arm are considered, namely biceps brachii (BIC), triceps brachii (TRI), flexor carpi radialis (FCR) and brachioradialis (BRD). The force, which is the output of the system, is acquired using a 6-DoF force/torque sensor (ATI Mini40, ATI Industrial Automation) mounted on the end effector of the robotic arm. For the model, we only consider the force component in the horizontal plane and perpendicular to the forearm of the subject. Real-time visual feedback of the force profile is provided to the user on a computer screen, in order to keep the applied forces within a maximum value of $\pm 20 \mathrm{~N}$.

Thanks to the aforementioned kinematic coupling, the coordinate of the elbow joint of the robot $(q)$ is acquired to 


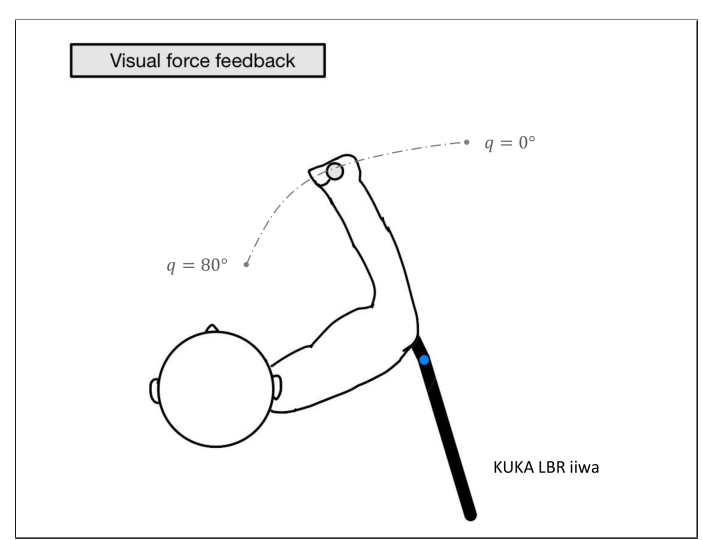

Fig. 1. Schematic representation of the acquisition setup: the user rests his arm on the KUKA robot while standing. EMG and force are synchronously acquired. The span of the scheduling signal $q$ is shown with a dash-dotted line. A computer screen in front of the user provides the visual force feedback in real time.

be used as the scheduling signal of the LPV model. The Fast Research Interface (FRI) [24] of the KUKA robot is exploited in order to acquire the force and scheduling signals at $100 \mathrm{~Hz}$.

The study population consists in two male subjects with an average age of 35.5 years and an average Body Mass Index (BMI) of $24.5 \mathrm{~kg} / \mathrm{m}^{2}$.

\section{A. EMG Processing}

The so-called muscular activation signal is derived from the raw EMG data for each muscle according to the following processing steps. The acquisition system applies a first bandpass filter between 20 and $450 \mathrm{~Hz}$, in order to filter out offsets and baseline drifts as well as to avoid aliasing. Then, during the offline processing, the bandwidth of the signal is further reduced using a Butterworth filter that selects the frequencies between 20 and $350 \mathrm{~Hz}$, implemented by means of the series of a fifth order high-pass and a second order low-pass. Full-Wave Rectification is later applied to the resulting signal by taking its absolute value. The last filtering step is the envelope extraction, which is performed using a second order Butterworth low-pass filter with a cut-off frequency of $1.775 \mathrm{~Hz}$. Starting from state-of-the-art values, the parameters of the filtering stages have been optimized in order to get the highest modeling accuracy.

\section{IDENTIFICATION OF THE EMG-ForCE RELATIONSHIP}

In this Section, the two LPV identification approaches exploited to obtain the model of the 1-DoF arm are described. The results of model validation have been computed by comparing the estimated force output with the measured signals in terms of goodness of FIT [\%] and Variance Accounted For (VAF) [\%].

\section{A. Local Identification Approach}

The basic idea of the local approach for LPV modeling is to consider the nonlinear, position-dependent system as a set of Linear Time-Invariant (LTI) models for different working points of the scheduling signal $q$. Hence, a successful description of the EMG-Force relationship at constant values of $q$ by means of LTI models is essential to build the LPV model following this approach. Therefore, several identification experiments are performed at constant angular positions. Specifically, 8 local datasets are acquired at $q=10,20,25,30,40,45,50,60\left[^{\circ}\right]$. Considering the human elbow angle, $q=-40^{\circ}$ corresponds to the full extension of the arm (i.e. $180^{\circ}$ ).

The local LTI models are identified at each of these angular positions using numerical algorithms for state-space models based on sub-space identification (N4SID) [19]. The identification is done in free form with prediction focus, thus minimizing the 1 -step ahead prediction error.

1) Local LTI Models: The average estimation performance of the LTI models on the local datasets resulted in $87.75 \%$ of FIT and $98.32 \%$ of VAF. It is interesting to evaluate the accuracy of these local models as a function of the distance $\Delta q=q_{I}-q_{V}$ between the identification position $q_{I}$ and the validation position $q_{V}$. More precisely, the LTI model identified at $q_{I}=20^{\circ}$ is validated using the datasets acquired at $q_{V}=10,40,60\left[^{\circ}\right]$. As shown in Fig. 2, the performance of the LTI model drops as $\Delta q$ increases. In particular, the FIT is much more sensitive to the angular distance. The accuracy of the local linear models is acceptable for low displacements from the identification position, while it decreases dramatically for $\Delta q>35^{\circ}$. This corroborates the modeling hypothesis of a positiondependent system: the EMG-Force relationship does depend
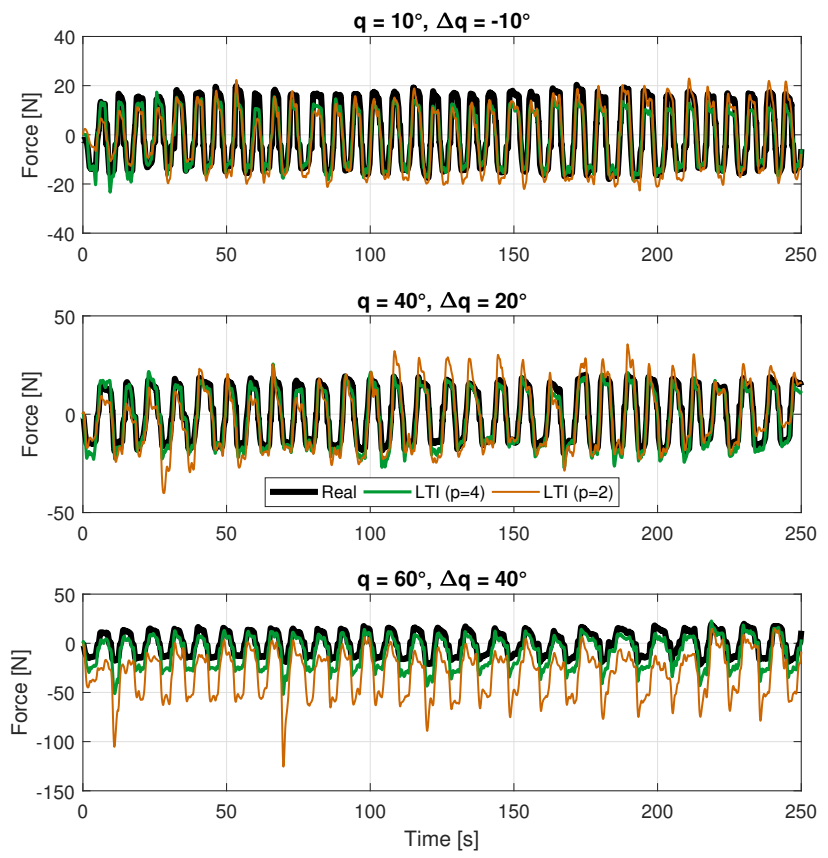

Fig. 2. Force plots comparing the real output (solid black) with the estimated output of the LTI model identified at $q=20^{\circ}$ and validated at $q=10^{\circ}$ (top panel), $q=40^{\circ}$ (middle panel) and $q=60^{\circ}$ (bottom panel). In the plots, the green line corresponds to the LTI model identified with $p=4$ inputs, while the orange line refers to LTI models identified from the signals of biceps and triceps only $(p=2)$. 




Fig. 3. Validation of the locally-identified LPV model on a local trial (acquired at $q=40^{\circ}$ ). The measured force (solid black) is compared to the signal estimated by the LPV model (solid green).

on the elbow angle.

2) Locally-identified LPV Model: The sub-space method used for the identification of the local LTI models may have produced LTI state-space models belonging to different bases. Hence, each LTI state-space model must be transformed to the same basis before the interpolation, in order for this to make sense. In this work, a Gramian-based balancing transformation of the state-space realizations is used [25, 26]. After this, the coefficients of the ALPV model are estimated by means of the Levenberg-Marquardt nonlinear least-squares algorithm.

The LPV model obtained by means of the Local Identification Framework (denoted as LIF-LPV) can be validated with a twofold approach. On the one hand, the local dataset acquired at $q=40^{\circ}$ is used to evaluate the accuracy of the LPV model obtained by interpolation. On the other hand, it is more significant to perform model validation considering global trials, as they describe a more realistic scenario.

In the former case, the average FIT of the LIF-LPV model is equal to $84.32 \%$ and its average VAF is equal to $97.80 \%$. On the local trials, the validation performance of the LPV model is very similar to the estimation performance of the local LTI models, which consists in an average FIT equal to $85.83 \%$ and an average VAF equal to $98.02 \%$. On the other hand, in the latter case, the validation performance of the LIF-LPV model decreases considerably. In particular, the average FIT is $74.10 \%$ and the average VAF is $95.43 \%$, as reported in Table I. It can be noticed a different behavior of the two indicators: while the average VAF of the LIFLPV model remains close to the performance obtained during local validation, the FIT drops significantly.

\section{B. Global Identification Approach}

The Global Identification Framework for LPV modeling is carried out exploiting the Prediction-Based Subspace Identification (PBSID) toolbox [27] and the LPVcore toolbox [20], both developed for MATLAB. The output of the PBSID toolbox is used to initialize the computation of the LPVcore toolbox. This allows to refine the estimation of the LPV model identified globally. This algorithm identifies the statespace LPV model by means of a gradient-based optimization technique. The LPV model obtained by means of the Global
Identification Framework (denoted as GIF-LPV) is again a discrete-time, second order, ALPV model.

For each user, the acquisition of a global dataset is repeated four times. In this way, one of these datasets is used to identify the LPV model according to the global approach, while the other three are used to perform model validation.

The average FIT and VAF of the GIF-LPV model are $78.25 \%$ and $95.66 \%$, respectively. As expected, the global identification approach for LPV modeling gives better performance with respect to the local one considering globaltrial validation. This is due to the different datasets used for the identification of the LPV model according to the two approaches. In particular, the GIF-LPV model is identified using a global dataset containing information about the arm dynamics during time variations of the elbow angle. On the other hand, this information is not contained in the localtrial datasets, as the arm is in a fixed position. Moreover, it should be noted that, while the performance of the LIFLPV model decreased when validated on a global trial, the GIF-LPV model performs well also if validated on datasets obtained with local trials.

\section{Performance Comparison}

In this Section, the performance of the LPV model are compared to the results obtained by a Hammerstein model, which is identified as a reference term for the LPV approach. Moreover, it is interesting to evaluate the validation performance on global trials of the LTI approach, given its accuracy in the case of constant-posture trials (see §IV-A.1).

The Hammerstein model is identified using the System Identification Toolbox of MATLAB. On the other hand, the LTI model (hereafter, globally-identified LTI or gLTI for brevity) is identified using again the N4SID algorithm. For both models, the identification dataset is the same global trial used also in the case of the GIF-LPV model.

The performance of the globally-identified LTI model are presented first. The gLTI model outperforms the LPV model identified following the local approach (LIF-LPV), with an average FIT of $76.75 \%$ and an average VAF of $94.75 \%$.
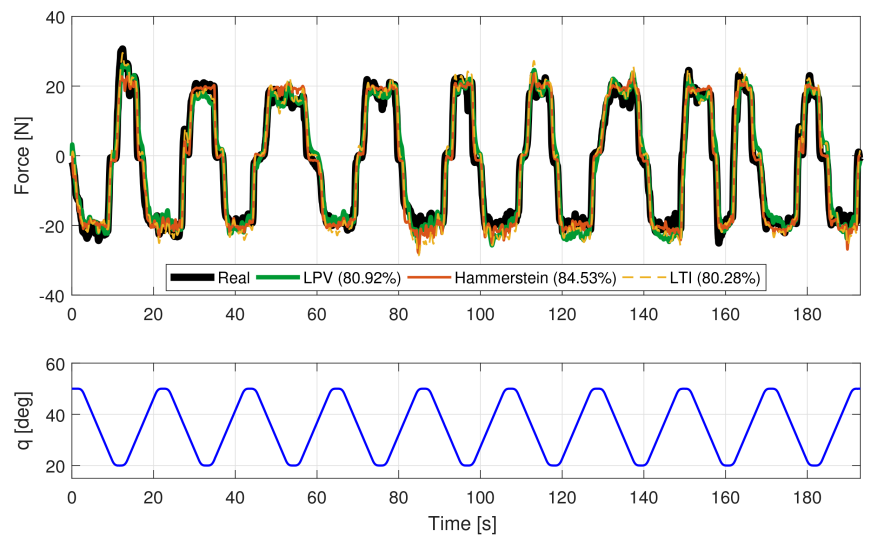

Fig. 4. Validation force plot comparing the GIF-LPV model with the Hammerstein model and the globally-identified LTI model. The scheduling variable trajectory $q(t)$ is shown in the bottom panel of the plot. 
TABLE I

VALIDATION RESULTS COMPARING THE PERFORMANCE ON GLOBAL TRIALS OF LTI, LIF-LPV AND GIF-LPV. THE PERFORMANCE OF THE

HAMMERSTEIN MODEL ARE REPORTED AS REFERENCE TERM.

\begin{tabular}{|c|c|c|c|c|c|}
\cline { 3 - 6 } \multicolumn{2}{c|}{} & LTI & LIF-LPV & GIF-LPV & Hammerstein \\
\hline \multirow{2}{*}{ User 1 } & FIT [\%] & 72.61 & 67.77 & 75.37 & 80.12 \\
\cline { 2 - 6 } & VAF [\%] & 93.12 & 94.63 & 94.88 & 96.19 \\
\hline \hline \multirow{2}{*}{ User 2 } & FIT [\%] & 80.89 & 80.42 & 81.12 & 85.76 \\
\cline { 2 - 6 } & VAF [\%] & 96.38 & 96.22 & 96.44 & 97.98 \\
\hline \hline \multirow{2}{*}{ Average } & FIT [\%] & 76.75 & 74.10 & 78.25 & 82.94 \\
\cline { 2 - 6 } & VAF [\%] & 94.75 & 95.55 & 95.66 & 97.09 \\
\hline
\end{tabular}

Although this result may sound surprising, the reason behind it is twofold. The first one is the difference between the datasets used to identify the two models. In particular, the LIF-LPV model is identified using a batch of local-trial datasets, in which the scheduling signal is constant and the muscles contract in isometric conditions. On the other hand, the LTI model is in this case identified on a global trial, thus being able to describe the dynamics of the system due to the time variations of the scheduling signal and to the non-isometric muscular contractions. The second reason is what we call here the linearization effect actuated by the muscles of the forearm. These muscles (BRD and FCR) are not included in the models of the 1-DoF arm presented in the literature. Indeed, their effect is generally neglected as the wrist motion is externally constrained. On the other hand, they have a non-negligible role in the process of endpoint force production considering the 1-DoF arm. Moreover, their EMG-Force relationship is not influenced by the elbow angle. Consequently, their contribution helps reducing the overall nonlinearity of the system. This is confirmed by evaluating the performance of the LTI model identified using the activation signals of biceps and triceps only (i.e. with $p=2$ inputs) instead of using the full set of input signals (i.e. $p=4$ ). In the former case, the linear model is much more sensitive to the elbow joint coordinate and hence to $\Delta q$. This is evident comparing the force signals in Fig. 2.

The results of the Hammerstein model are presented at last. This modeling approach scores an average FIT of $82.94 \%$ and an average VAF of $97.09 \%$ on global trials. As expected, the nonlinear model outperforms both the LTI and the LPV models, allowing the highest modeling accuracy. The validation results on global trials of all the presented models are summarized in Table I.

\section{DISCUSSION}

The first interesting result presented in this work is that local, linear models (LTI) are accurate enough to describe the EMG-Force relationship for constant-posture trials. This demonstrated the feasibility of modeling the human arm exploiting the Local Identification Framework for LPV models. Although feasible, the performance in terms of FIT decreased dramatically considering global validation trials instead of constant-posture trials. As argued above, this increase of the estimation error is due to the lack of information about the time-varying dynamics of the elbow angle in the local datasets, which also results in non-isometric muscular contractions. This is the main limitation of the local approach for LPV identification. Indeed, the behavior of the true LPV system cannot be fully determined interpolating a set of local models identified at several values of the scheduling signal, as the system is sensitive not only to $q$, but also to its time variations. Aside to this intrinsic limitation of the local identification approach, also the behavior of the muscles changes from local to global trials. Indeed, the constantposture constraint is released in the latter case. Therefore, the muscles work in isometric conditions in the case of the identification trials and in non-isometric conditions in the case of the validation trials. Hence, the more complex dynamics of the validation data cannot be described by the LIF-LPV model, since it is not contained in the dataset used for its identification.

As expected, the LPV model identified globally (GIFLPV) outperforms both the LIF-LPV and the gLTI. Nevertheless, the Global Identification Framework is generally a less practical choice compared to the Local Identification Framework for LPV modeling. Moreover, the global identification of LPV models is still under ongoing research, affecting especially the development of algorithms and tools for the identification process itself. Consequently, the performance of this approach is expected to increase in the near future.

Another important result presented in this work is the importance of including the muscles of the forearm, namely the brachioradialis and the flexor carpi radialis, while releasing the external constraints on the wrist movement. Indeed, these muscles have an important role in force generation. This is mainly due to the unconscious, synchronous activity they manifest while biceps and triceps are consciously activated when the subjects interact with the robot. This may be partially related to the fact that the wrist was not constrained during the acquisitions. One could argue that the identified model actually describes the quasi-2-DoF arm, because of the activation of the muscles of the forearm actuating the wrist. Nevertheless, the aim of the identified model is to provide a sufficiently accurate estimation of the force produced by the human arm considering an experimental setup similar to a real-case scenario of HRI. As a consequence, it was chosen to let the wrist free from external constraints. As shown in this work, this aim has been achieved. The muscles of the forearm, in fact, induce a linearization effect of the EMG-Force relationship, thus allowing higher accuracy with 
a lower complexity modeling approach.

\section{CONCLUSION AND FUTURE DEVELOPMENTS}

In this work, we have addressed the problem of modeling the EMG-Force relationship of the 1-DoF human arm with a low-complexity, control-oriented approach, by means of Linear Parameter Varying (LPV) models. These results show that an LPV model can be a good compromise in terms of accuracy and complexity with respect to state-of-theart alternatives. Releasing the external constraints off the wrist and including the forearm muscles appeared to be fundamental to achieve such accurate results. The reported performance metrics legitimate this approach, that can be further investigated with the aim of integrating the identified model into the control system of collaborative robots.

For the future, several improvements to the experimental setup could be done. First of all, the number of subjects of the study could be increased in order to have statistically significant results. Moreover, it could be useful to have a direct measurement of the elbow angle of the human subject. This could allow to investigate also different interaction scenarios between the robot and the human operator, as the kinematic coupling would be no more necessary. Finally, the model could be extended adding the degree of freedom due to the actuation of the wrist and/or the shoulder.

\section{ACKNOWLEDGMENTS}

This work has been published under the framework of the IdEx Unistra and benefits from a funding from the State managed by the French National Research Agency as a part of the Investments for the future program.

\section{REFERENCES}

[1] J.-Y. Hogrel. "Clinical applications of surface electromyography in neuromuscular disorders". In: Neurophysiologie Clinique/Clinical Neurophysiology 35.2-3 (), pp. 59-71.

[2] R. Bogue. "Rehabilitation robots". In: Industrial Robot: An International Journal 45.3 (2018), pp. 301-306.

[3] L. M. Weber and J. Stein. "The use of robots in stroke rehabilitation: A narrative review". In: NeuroRehabilitation 43.1 (2018), pp. 99-110.

[4] L. E. Kahn et al. "Robot-assisted reaching exercise promotes arm movement recovery in chronic hemiparetic stroke: a randomized controlled pilot study". In: Journal of neuroengineering and rehabilitation 3.1 (2006), p. 12.

[5] A. Basteris et al. "Training modalities in robot-mediated upper limb rehabilitation in stroke: a framework for classification based on a systematic review". In: Journal of neuroengineering and rehabilitation 11.1 (2014), p. 111.

[6] A. V. Hill. "The heat of shortening and the dynamic constants of muscle". In: Proc. R. Soc. Lond. B 126.843 (1938), pp. 136-195.

[7] D. Buongiorno et al. "A linear approach to optimize an EMG-driven neuromusculoskeletal model for movement intention detection in myo-control: A case study on shoulder and elbow joints". In: Frontiers in neurorobotics 12 (2018), p. 74.

[8] E. A. Clancy et al. "Identification of constant-posture EMGtorque relationship about the elbow using nonlinear dynamic models". In: IEEE Transactions on Biomedical Engineering 59.1 (2012), pp. 205-212.
[9] J. Hashemi et al. "EMG-force modeling using parallel cascade identification”. In: Journal of Electromyography and Kinesiology 22.3 (2012), pp. 469-477.

[10] J. Hashemi et al. "Enhanced dynamic EMG-force estimation through calibration and PCI modeling". In: IEEE Transactions on Neural Systems and Rehabilitation Engineering 23.1 (2015), pp. 41-50.

[11] P. Liu et al. "Influence of joint angle on EMG-torque model during constant-posture, quasi-constant-torque contractions". In: Journal of Electromyography and Kinesiology 23.5 (2013), pp. 1020-1028.

[12] S. van Eesbeek et al. "LPV subspace identification of timevariant joint impedance". In: Neural Engineering (NER), 2013 6th International IEEE/EMBS Conference on. IEEE. 2013, pp. 343-346.

[13] M. A. Golkar, E. Sobhani Tehrani, and R. E. Kearney. "Linear Parameter Varying Identification of Dynamic Joint Stiffness during Time-Varying Voluntary Contractions". In: Frontiers in computational neuroscience 11 (2017), p. 35.

[14] M. Petreczky, R. Tóth, and G. Mercre. Realization theory for LPV state-space representations with affine dependence. 2017. DOI: 10.1109/TAC.2016.2629989.

[15] Z. Alkhoury, M. Petreczky, and G. Mercère. "Identifiability of affine linear parameter-varying models". In: Automatica 80 (2017), pp. $62-74$.

[16] J. Mohammadpour and C. Scherer. Control of Linear Parameter Varying Systems with Applications. New York: Springer, 2012.

[17] O. Sename, P. Gáspár, and J. Bokor. Robust control and linear parameter varying approaches, application to vehicle dynamics. Springer New York Dordrecht Heidelberg London, 2013.

[18] R. Tóth. Modeling and identification of linear parametervarying systems. Vol. 403. Springer, 2010.

[19] P. Van Overschee and B. De Moor. Subspace identification for linear systems: TheoryImplementationApplications. Springer Science \& Business Media, 2012.

[20] P. B. Cox, R. Tóth, and M. Petreczky. "Towards efficient maximum likelihood estimation of LPV-SS models". In: Automatica 97 (2018), pp. 392-403.

[21] M. Lovera and G. Mercère. "Identification for gain scheduling: a balanced subspace approach". In: Proceedings of the American Control Conference. New York, USA, 2007.

[22] Y Zhu and Z. Xu. "A method of LPV model identification for control". In: Proceedings of the IFAC World Congress 17 (2008), pp. 5018-5023. ISSN: 14746670. DOI: $10.3182 /$ $20080706-5-\mathrm{KR}-1001.4064$.

[23] Z. Alkhoury, M. Petreczky, and G. Mercère. "Comparing global input-output behavior of frozen-equivalent LPV state-space models". In: IFAC-PapersOnLine 50.1 (2017), pp. 9766-9771.

[24] G. Schreiber, A. Stemmer, and R. Bischoff. "The fast research interface for the kuka lightweight robot". In: IEEE Workshop on Innovative Robot Control Architectures for Demanding (Research) Applications How to Modify and Enhance Commercial Controllers (ICRA 2010). Citeseer. 2010, pp. 15-21.

[25] A. Laub et al. "Computation of system balancing transformations and other applications of simultaneous diagonalization algorithms". In: IEEE Transactions on Automatic Control 32.2 (1987), pp. 115-122.

[26] B. Moore. "Principal component analysis in linear systems: Controllability, observability, and model reduction". In: IEEE transactions on automatic control 26.1 (1981), pp. 17-32.

[27] J.-W. Van Wingerden and M. Verhaegen. "Subspace identification of bilinear and LPV systems for open-and closed-loop data". In: Automatica 45.2 (2009), pp. 372-381. 